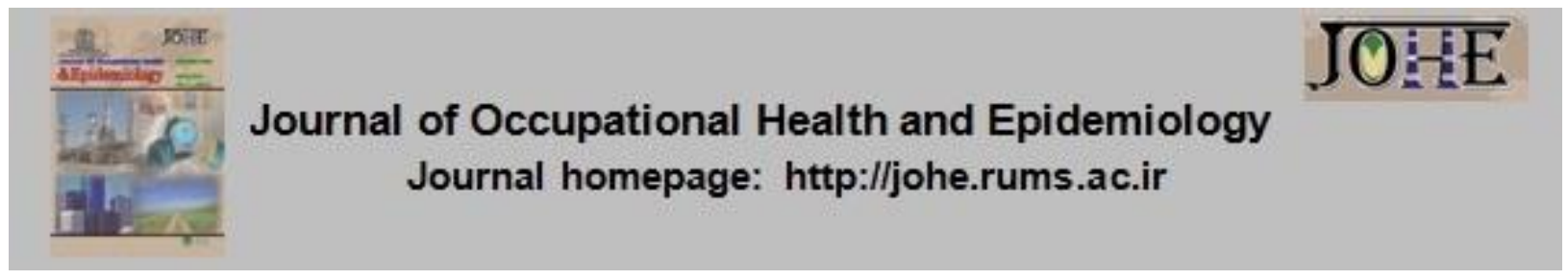

\title{
Validity and reliability of the Chester step test for prediction of the aerobic capacity among Iranian students
}

\author{
Mahnaz Saremi ${ }^{1,}{ }^{*}$, Fatemeh Khayati ${ }^{2}$, Fatemeh Mousavi ${ }^{2}$ \\ 1- Associate Prof., Department of Ergonomics, School of Health, Safety and Environment, Shahid Beheshti \\ University of Medical Sciences, Tehran, Iran. \\ 2- MSc in Ergonomics, School of Health, Safety and Environment, Shahid Beheshti University of Medical Sciences, \\ Tehran, Iran.
}

\section{Article Info}

* Corresponding authors:

Mahnaz Saremi,

E-mail:

m.saremi@sbmu.ac.ir

\section{Article history}

Received: Dec, 2017

Accepted: Feb, 2018

Print ISSN: 2251-8096

Online ISSN: 2252-0902

Peer review under responsibility of Journal of Occupational Health and Epidemiology

\begin{abstract}
Background: Assessment of aerobic capacity is of vital importance in both general community and occupational settings. Valid, reliable and feasible tests are required to indicate the functionality of cardiopulmonary system. The present study was carried out aiming to evaluate the suitability of the Chester step test (CST) as a field test to measure the Iranians' aerobic capacity.

Materials and Methods: In this descriptive study performed in 2016, 63 university students completed the Astrand-rhyming cycle ergometer test and the CST. Actual and predicted maximum rate of oxygen consumption $\left(\mathrm{VO}_{2}\right.$ max) were recorded and their relationship was examined by Pearson's correlation coefficient and Bland-Altman plot. Repeatability was checked using intra-class correlation coefficient (ICC).

Results: Predicted and actual amounts of $\mathrm{VO}_{2}$ max were significantly correlated $(r=0.868, P<0.001)$. The mean difference between them was $0.89 \mathrm{ml} / \mathrm{kg} / \mathrm{minute}$. ICC between CST and the retest CST (CST retest $_{\text {) was }}$ 0.858 .

Conclusions: CST is a valid and reliable field test for the estimation of cardiorespiratory capacity among the study subjects. With its exclusive features (e.g. safety, convenience and ease of use), CST could be suggested as an alternative to other existing methods of estimating $\mathrm{VO}_{2}$ max; the applicability of the existing methods among Iranian individuals are unclear. However, like other estimation methods, special prudence should be adopted when dealing with critical health decisions.
\end{abstract}

Keywords: Validity, Students, Iran

\section{Introduction}

Despite the progressive automation trend, there is still a huge need for human physical force in many industries, especially in developing countries (1). Most of these works demand high physical efforts and exertions. The fundamental principle of ergonomics is to fit the job to the worker (2). In the physical ergonomics domain, this means that the physical demand of work should not exceed the physical work capacity (PWC). Therefore, a suitable level of employees' health, safety, efficiency, motivation and, satisfaction can be achieved (3).

Therefore, the proper assessment of physical work capacity is essential in many working situations. The maximum oxygen

(C) The Author(s) 2018. Published by Rafsanjan University of Medical Sciences Press.

All rights reserved. For permissions, please e-mail: johe.rums@gmail.com, johe@rums.ac.ir 
uptake and also the cardiovascular capacity are the most well known indices for determining a human energy expenditure (4). In fact, a massive body of knowledge supports that the maximum volume of oxygen $\left(\mathrm{VO}_{2} \max \right)$ is the most reliable indicator for determining cardio-respiratory capacity (5). However, since direct measurement of $\mathrm{VO}_{2}$ max may expose some people (i.e. those who have not a high degree of cardio-respiratory ability) at risk, the prediction methods were proposed (4, $5)$. Being highly dependent on laboratory equipment, exhausting and difficult experimental protocol, need to expert operator and difficult training, being expensive and time consuming are also among the most frequent cited problems attributed to the maximal tests $(6,7)$. It is therefore desirable to use simple yet valid procedures for evaluating $\mathrm{VO}_{2}$ max, especially when a well-equipped laboratory is absent.

The prediction methods are based on the fact that heart rate increases as a function of workload and oxygen consumption. Therefore, since it is more easily measured compared to oxygen consumption, heart rate is usually used as an indirect measurement of energy expenditure $(4,5)$.

Numerous sub-maximal protocols have emerged for this purpose (8-11), among them, the Chester step test (CST) is one of the simplest protocols. Kevin Sykes developed CST at Chester university of United Kingdom. This test was designed to provide a safe and practical procedure for assessing aerobic fitness under submaximal conditions $(7,12)$. CST is also claimed to be inexpensive, safe, portable and highly applicable and adaptable to a wide range of age and fitness (7). This test was already applied for determination of aerobic fitness of fire brigades in British, European, north American and Asian workers $(7,12)$, disaster personnel (13) and children (14). However, its applicability has not yet been explored among Iranian population.

Aerobic capacity could be influenced by many factors including age, body weight, race, lifestyle, sport, alcohol and tobacco consumption, nutritional status, motivation and physiological parameters $(15,16)$. Moreover, in step test protocols, the energy required to climb each step could be different as a function of each person's leg length. This argument led Sykes and Roberts to consider various step heights for CST in order to develop a more accurate step protocol for estimating the aerobic capacity (7).

To date, no studies have attempted to evaluate the suitability of the CST for application among the Iranian population. The present study was conducted aiming to assess the validity and reliability of CST to predict $\mathrm{VO}_{2}$ max among the Iranian adults.

\section{Material and Methods}

This cross-sectional experiment was conducted in the ergonomics laboratory of the school of Health, Safety and Environment of Shahid Beheshti University of Medical Sciences, Iran, in 2016. The sample size was determined based on the previous similar studies. Buckley et al. recruited 13 students for examining validity and reliability of the CST (12), while Sykes and Roberts used 68 subjects (7). In the same way and respecting the voluntarily participation, university students were recruited by announcement. Subjects suffering from any musculoskeletal disorders, cardiopulmonary diseases or with exercise prohibition were excluded. Finally, 63 students including 29 women and 34 men participated in the study. The study was conducted with the approval of the local ethics committee with the code N. 16712/24. A signed written informed consent was received from all the subjects.

Indoor air temperature was set at $22{ }^{\circ} \mathrm{C}$. Subjects were asked to eat a light breakfast 
2 hours prior the test session and to refrain afterwards from eating, drinking caffeine or smoking until the end of the trial. They were also requested to avoid exercise for 24 hours before the trial.

The experiment included 2 sessions of oxygen uptake measurement (i.e. an ergospirometry test and a step test, described below), conducted on two separate days interspersed by one week interval in order to avoid any residual effect of fatigue. Again, the step test was repeated after a one-week recovery, called $\mathrm{CST}_{\text {retest }}$. All measurements were taken by one experienced investigator between 9:00 and 12:00 A.M. Before starting each session, subjects were requested to rest a few minutes in order to reach their baseline metabolism (17). Individual factors of age, height, and weight were recorded for further analysis. Body mass index (BMI) was calculated in $\mathrm{kg} / \mathrm{m}^{2}$.

Following the Astrand and Rhyming 6minute protocol (8), $\mathrm{VO}_{2}$ max was measured on an electronic bicycle ergometer (Monark Ergomedic 839E, Sweden) equipped with a gas analyzer system (breath by breath Metalyzer 3B, Cortex Biophysics, Germany). The protocol was designed to determine maximal oxygen consumed by the subject during exercising at a submaximal workload. Manually set during the first two minutes of the test, the workload was maintained difficult enough to elicit a steady heart rate of at least 120 (beats per minute) bpm. During the test session, the workload was progressively increased until the heart rate varied no more than $4 \mathrm{bpm}$. Before starting the test, subjects were familiarized by pedaling during a 2-minute warm-up period (18). Expired gasses [carbon dioxide $\left(\mathrm{CO}_{2}\right)$ and Oxygen $\left(\mathrm{O}_{2}\right)$ ] and heart rate were continually monitored. The test was immediately interrupted once the subject rated his/her perceived exertion at or above 15 on Borg's 6-20 scale or reached at his/her maximum age-related heart rate (220-age) (18).

The step test procedure was conducted based on the CST general guidelines.

A pre-recorded audiotape served as metronome to regulate stepping rhythms. After a short briefing, the CST was run while the subject synchronized his/her steps with metronome beats at progressive rates of 15 , $20,25,30$, and 35 steps per minute for each consecutive two minutes. Heart rate was monitored continuously during the test by means of a beltless pulse monitor (Beurer $\mathrm{GmbH} \&$ Co, Germany). At the end of each stage, heart rate and rating of perceived exertion (RPE) were recorded. As the stop rule of the test indicated, the procedure continued as long as the subject showed no signs of over exhaustion (RPE $<15$ on Borg's 6-20 scale) or heart rate not above $85 \%$ of age-related maximum. The entire duration of the test was 10 minutes. $\mathrm{VO}_{2}$ max was predicted by drawing the "line of best fit" on the CST graphical datasheet (7). The data normal distribution was assessed by the Shapiro-Wilk test. Pearson's correlation coefficient was used for determining the relation between quantitative data derived from ergospirometry and CST. Paired t-test was used to compare the test-retest variability of predicted $\mathrm{VO}_{2}$ max between CST and $\mathrm{CST}_{\text {retest }}$. The reproducibility of $\mathrm{VO}_{2}$ max by CST was determined using the intra-class correlation coefficient (ICC). The BlandAltman plot was employed to measure the agreement between the two methods. To construct a Bland-Altman plot, the difference between CST with the ergospiratory test was plotted on the $y$-axis against the average of the total amount on the $x$-axis (19). The level of significance was set at 0.050 for all analyses. Data were analyzed using SPSS (version 20, IBM Corporation, Armonk, NY, USA) and MedCalc statistical software. 


\section{Results}

Sociodemographic features and aerobic capacity of subjects are summarized by sex in table 1 . The mean age of subjects was $20.17 \pm 1.80$ years. Their age, height, weight and $\mathrm{BMI}$ ranged from 18 to 29 years, 152 to $193 \mathrm{~cm}, 46$ to $130 \mathrm{~kg}$, and 16.6 to $36.5 \mathrm{~kg} / \mathrm{m}^{2}$, respectively. The level of fitness varied from 31 to 58 ( $\mathrm{ml} / \mathrm{kg} /$ minute).

Table 1: Mean (SD) of sociodemographic features and aerobic capacity of the Iranian students (2016)

\begin{tabular}{|c|c|c|c|c|c|c|c|c|}
\hline Subject & $\mathbf{n}$ & $\begin{array}{c}\text { Age } \\
\text { (year) }\end{array}$ & $\begin{array}{c}\text { Wight } \\
\text { (kg) }\end{array}$ & $\begin{array}{l}\text { Height } \\
\text { (cm) }\end{array}$ & $\begin{array}{c}\mathrm{BMI}^{*} \\
\left(\mathrm{Kg} / \mathrm{m}^{2}\right)\end{array}$ & $\begin{array}{c}\text { Ergo- } \\
\text { spirometry }\end{array}$ & CST ** & $\mathbf{C S T}_{\text {retest }}$ \\
\hline Male & 29 & $\begin{array}{l}19.34 \\
(1.47)\end{array}$ & $\begin{array}{c}75.24 \\
(18.02)\end{array}$ & $\begin{array}{c}180.62 \\
(4.41)\end{array}$ & $\begin{array}{l}23.02 \\
(5.23)\end{array}$ & $43.67(6.55)$ & $\begin{array}{l}44.50 \\
(6.93)\end{array}$ & $\begin{array}{l}44.13 \\
(5.81)\end{array}$ \\
\hline Female & 34 & $\begin{array}{l}20.88 \\
(1.88)\end{array}$ & $\begin{array}{c}58.88 \\
(12.22)\end{array}$ & $\begin{array}{c}164.44 \\
(5.98)\end{array}$ & $\begin{array}{l}21.72 \\
(5.27)\end{array}$ & $37.48(4.94)$ & $\begin{array}{l}38.61 \\
(5.30)\end{array}$ & $\begin{array}{l}35.57 \\
(5.09)\end{array}$ \\
\hline Total & 63 & $\begin{array}{l}20.17 \\
(1.86)\end{array}$ & $\begin{array}{c}66.14 \\
(17.08)\end{array}$ & $\begin{array}{c}171.88 \\
(9.69)\end{array}$ & $\begin{array}{l}22.27 \\
(4.44)\end{array}$ & $40.51(6.52)$ & $\begin{array}{l}41.87 \\
(7.55)\end{array}$ & $\begin{array}{l}40.13 \\
(6.89)\end{array}$ \\
\hline
\end{tabular}

* BMI: Body mass index

** CST: Chester step test; Measured in $\mathrm{ml} \mathrm{O}_{2} / \mathrm{kg} /$ minute

$\mathrm{VO}_{2}$ max was different as a function of sex and BMI $(P<0.050$, for both cases). This rate was significantly higher among men compared to women with $43.67 \pm 6.51$ and $37.48 \pm 4.92 \mathrm{ml} / \mathrm{kg} /$ minute, respectively $(P=$ $0.001)$, and decreased with BMI increasing $(P=0.003)$.
A rather strong correlation $(r=0.868, P<$ $0.001)$ was found between the results obtained from ergospirometry and CST, which was marginally higher among men $(r$ $=0.874, P<0.001)$ in comparison with women $(r=0.803, P<0.001)$.

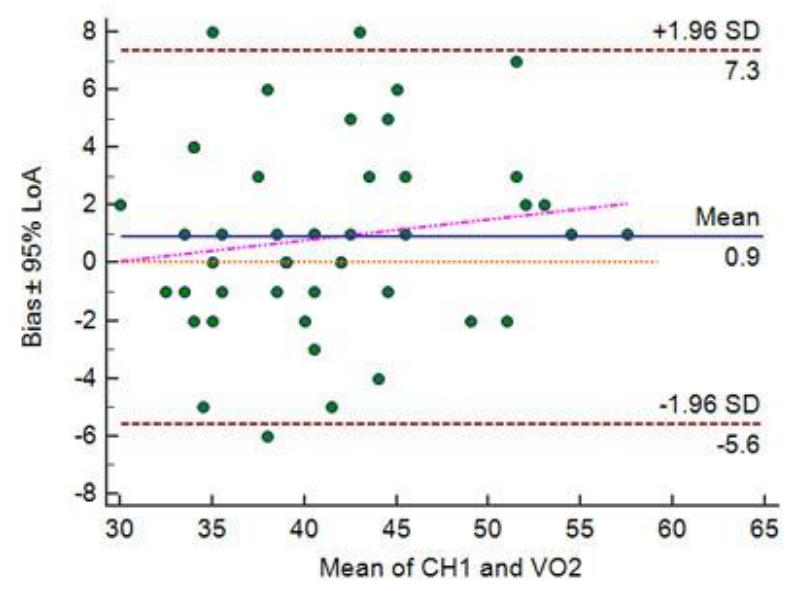

Figure 1: Limit of agreement of confidence interval of $95 \%$ for aerobic capacity measurements in $\mathrm{ml} / \mathrm{kg} /$ minute between ergospirometry and Chester step test (CST)

According to the Bland-Althman method, mean difference (bias) of actual and predicted $\mathrm{VO}_{2}$ max and $95 \%$ limit of agreement (average difference \pm 1.96 standard deviation of the difference) were $0.89(S D=3.29)$ and 6.45 , respectively.
According to figure 1, the bias between the actual and predicted $\mathrm{VO}_{2}$ max was not statistically significant $(P=0.073)$, meaning that the differences between mean \pm 1.96 SD were not clinically important and thus the two methods of ergospirometry and CST 
could be considered in agreement and may be used interchangeably.

The statistical difference between CST and $\mathrm{CST}_{\text {retest }}$ with mean $\pm \mathrm{SD}$ of respectively $41.87 \pm 7.55$ and $40.13 \pm 6.89 \mathrm{ml} / \mathrm{kg} /$ minute, was not significant $(P=0.209)$. ICC between CST and CST retest was 0.858 [95\% confidence interval $(\mathrm{Cl}), 0.41$ to 0.91$]$, indicating a high test-retest reliability.

\section{Discussion}

Despite numerous studies related to the sub-maximal tests, there is still need for confirming the reliability and validity of such tests for specific populations $(17,20)$. In the present study, the high correlation found between actual and predicted $\mathrm{VO}_{2} \max$ confirmed the applicability of CST among the study subjects both for men and women. CST was previously found as a valid method for prediction of $\mathrm{VO}_{2}$ max among the British adults (7). With an error margin of 5-15 percent, CST was declared as an accurate reflection of an individual's aerobic capacity (7). Buckley et al. questionned the validity of CST to predict actual $\mathrm{VO}_{2} \max$ due to a greater underestimation error of $19 \%$ observed in this study, however, the overestimation error remained at the same level of the study by Sykes and Roberts (12). As explained in this study, part of this swollen error prediction could due to the fact that the stepping procedures measure $\mathrm{VO}_{2}$ max by about $97 \%$ of the amount assessed by treadmill. However, the present study revealed that with a small bias of approximately 0.9 , CST is accurate enough to be used confidently rather than the direct measurement of $\mathrm{VO}_{2} \max (12)$.

Step tests are known as reliable tools for predicting the aerobic capacity $(7,10,12$, 17, 21). In accordance with previous studies, the reliability of CST was approved by bias of less than $-0.8 \mathrm{ml} / \mathrm{kg} /$ minute $(7$, 12). A good reliability of CST was already found for both men and women in a wide range of age and fitness $(7,12)$. Moreover, according to a systematic review conducted by Bennett et al., CST is defined as the best tool of its kind for monitoring alterations in cardiorespiratory fitness due to its remarkable test-retest reliability (10). CST was also demonstrated to be able to differentiate the functional capacity of patients with chronic obstructive pulmunary disease (COPD) from healthy subjects (22, 23).

Some researchers have already predicted the aerobic capacity of various Iranian groups. Abdossaleh and ahmadi used two submaximal protocols to estimate maximum oxigen uptake of Iranian men university students (mean age $=22.6$ years) which was found to be 45.5 and $42.8 \mathrm{ml} / \mathrm{kg} /$ minute by shuttle run test and queen step test, respectively (24). More recently, Heydari et al. reported an average of $3.10 \pm 0.50$ $\mathrm{l} /$ minute of maximum energy consumption for Iranian medical emergencies students (25). Using a step test, Firoozeh et al. predicted that the mean $\mathrm{VO}_{2}$ max of Iranian firefighters was $36.20 \pm 3.30 \mathrm{ml} / \mathrm{kg} /$ minute (26). Comparing to other nations, the aerobic capacity of Indian students was found to be approximately 45 and 37 $\mathrm{ml} / \mathrm{kg} /$ minute for men and women, respectively (27). In line with the majority of similar studies, the results of the present study suggest that a mean $\mathrm{VO}_{2}$ max of about $40-45$ and $35-40 \mathrm{ml} / \mathrm{kg} /$ minute would be expected for men and women students, respectively. However, due to the lack of norms for Iranian population, $\mathrm{VO}_{2}$ max data could not clearly be compared or interpreted,hence further studies are needed to fill this gap.

The body fat percentage influences all activities involving body movement. Therefore, reporting relative terms of $\mathrm{VO}_{2}$ max in $\mathrm{ml} / \mathrm{kg} /$ minute is more meaningful than its absolute value in $\mathrm{l} /$ minute. Having a higher body fat percentage, lower hemoglobin rate and cardiac output compared to men, are among the most cited factors attributed to lower aerobic fitness 
observed among women (5). These arguments could explain the lower aerobic capacity of fat and men subjects in the present study.

Although the findings of the present study showed the suitability of CST for prediction of aerobic capacity among Iranian young individuals, it is always questionable if CST is also convenient for other Iranian groups of age, health status, etc. Therefore, further studies are necessary to examine these issues.

\section{Conclusion}

CST could be considered as a valid and reliable sub-maximal test for prediction of maximum aerobic capacity of Iranian youth. However, similar to other sub-maximal tests, the results need to be considered with prudence in the case of important job decisions. Otherwise, CST is likely to be well admissible for job health promotion and rehabilitation purpose.

\section{Acknowledgement}

This study was financially supported by the research deputy of Shahid Beheshti University of Medical Sciences with a tracking code of 5073. The authors would like to thank all the students participated in this study.

Conflict of interest: None declared.

\section{References}

1. Marras WS, Cutlip RG, Burt SE, Waters TR. National occupational research agenda (NORA) future directions in occupational musculoskeletal disorder health research. Appl Ergon 2009; 40(1):15-22.

2. Grandjean E, Kroemer KHE. Fitting the task to the human: a textbook of occupational ergonomics. $5^{\text {th }}$ ed. Boca Raton, Florida, United States: CRC Press; 1997.

3. Leka S, Jain A. Health impact of psychosocial hazards at work: an overview. $1^{\text {st }}$ ed. Geneva, Switzerland: World Health Organization; 2010.
4. Åstrand PO. Textbook of work physiology: physiological bases of exercise. $1^{\text {st }}$ ed. Champaign, United States: Human Kinetics; 2003.

5. Powers S, Howley E. Exercise physiology: Theory and application to fitness and performance. $9^{\text {th }}$ ed. New York, United States: McGraw-Hill Education; 2015.

6. Grant $\mathrm{S}$, Corbett $\mathrm{K}$, Amjad AM, Wilson J, Aitchison T. A comparison of methods of predicting maximum oxygen uptake. $\mathrm{Br} \mathrm{J}$ Sports Med 1995; 29(3):147-52.

7. Sykes K, Roberts A. The chester step testa simple yet effective tool for the prediction of aerobic capacity. Physiotherapy 2004; 90(4):183-8.

8. Åstrand $\mathrm{PO}$, Ryhming I. A nomogram for calculation of aerobic capacity (physical fitness) from pulse rate during sub-maximal work. J Appl Physiol 1954; 7(2):218-21.

9. Bayly WM, Gabel AA, Barr SA. Cardiovascular effects of submaximal aerobic training on a treadmill in Standardbred horses, using a standardized exercise test. Am J Vet Res 1983; 44(4):544-53.

10. Bennett H, Parfitt G, Davison K, Eston R. Validity of Submaximal Step Tests to estimate maximal oxygen uptake in healthy adults. Sports Med 2016; 46(5):737-50.

11. Ekblom Ö. Testing the validity of three submaximal ergometer tests for estimating maximal aerobic capacity in children. Acta Paediatr 2014; 103(5):559-63.

12. Buckley JP, Sim J, Eston RG, Hession R, Fox $R$. Reliability and validity of measures taken during the Chester step test to predict aerobic power and to prescribe aerobic exercise. Br J Sports Med 2004; 38(2):197205.

13. Molloy MS, Robertson CM, Ciottone GR. Chester Step Test as a reliable, reproducible method of assessing physical fitness of disaster deployment personnel. South Med J 2017; 110(8):494-6.

14. Maggio $A B R$, Vuistiner $P$, Crettenand $A$, Tabin R, Martin XE, Beghetti $M$, et al. Adapting the "Chester step test" to predict peak oxygen uptake in children. Swiss Med Wkly 2017; 147:w14435.

15. Punakallio $A$, Lindholm $H$, Luukkonen $R$, Lusa S. Lifestyle factors predicting changes in aerobic capacity of aging firefighters at 3 and 13-year follow-ups. J Occup Environ Med 2012; 54(9):1133-41. 
16. Firoozeh M, Saremi M, Kavousi A, Maleki A. Demographic and occupational determinants of the work ability of firemen. J Occup Health 2017; 59(1):81-7.

17. Chatterjee S, Chatterjee P, Mukherjee PS, Bandyopadhyay A. Validity of Queen's College step test for use with young Indian men. Br J Sports Med 2004; 38(3):289-91.

18. Cheevers A, Pettersen C. Åstrand Bike Test, 6 minute submaximal exercise test. $26^{\text {th }}$ ed. Amesterdam, Netherlands: Hogeschool van Amsterdam; 2007.

19. Bland JM, Altman DG. Measuring agreement in method comparison studies. Stat Methods Med Res 1999; 8(2):135-60.

20. Sheahan PJ, Nelson-Wong EJ, Fischer SL. A review of culturally adapted versions of the Oswestry Disability Index: the adaptation process, construct validity, test-retest reliability and internal consistency. Disabil Rehabil 2015; 37(25):2367-74.

21. Webb C, Vehrs PR, George JD, Hager R. Estimating VO2max using a personalized step test. Meas Phys Edu Exerc Sci 2014; 18(3):184-97.

22. Karloh M, Correa KS, Martins LQ, Araujo CL, Matte DL, Mayer AF. Chester step test: assessment of functional capacity and magnitude of cardiorespiratory response in patients with COPD and healthy subjects. Braz J Phys Ther 2013; 17(3):227-35.

23. de Camargo AA, Justino $\mathrm{T}$, de Andrade $\mathrm{CH}$, Malaguti C, Dal Corso S. Chester step test in patients with COPD: reliability and correlation with pulmonary function test results. Respir Care 2011; 56(7):995-1001.

24. Abdossaleh Z, AHmadi F. Assessment of the Validity of Queens Step Test for estimation maximum oxygen uptake (VO2 max). International Journal of Sport Studies 2013; 3(6):617-22.

25. Heydari $P$, Varmazyar S, Nikpey A, Safari Variani A, Jafarvand M. Step Test: a method for evaluating maximum oxygen consumption to determine the ability kind of work among students of medical emergencies. Electron Physician 2017; 9(3):4020-6.

26. Firoozeh M, Saremi M, Maleki A, Kavousi A. Investigation of maximal aerobic capacity and associated factors in firefighters. Iran Occupational Health 2015; 12(3):15-26.

27. Nabi Khushoo T, Rafiq N, Qayoom O. Assessment of cardiovascular fitness [VO2 max] among medical students by Queens College step test. International Journal of Biomedical and Advance Research 2015; 6(5):418-21. 